\title{
Sinusoidal phase modulating interferometer for real-time surface profile measurement
}

\author{
He Guotian ${ }^{1,2 *}$, Jiang Helun ${ }^{3}$, Tan Xingwen ${ }^{4}$ \\ ${ }^{1}$ Chongqing University, Chongqing, China 400030 \\ ${ }^{2}$ College of Physics and Information Technology, Chongqing Normal University, Chongqing, \\ China 400047 \\ ${ }^{3}$ Chongqing Technology and Business University, Chongqing, China 400067 \\ ${ }^{4}$ Southwest Universry, Chongqing, China 400700 \\ *Corresponding author: slhgt@siom.ac.cn
}

\begin{abstract}
The optical interferometry for the surface profile measurement is high accuracy, non-contact, and has a wide application in industry and scientific research. In this paper, a sinusoidal phase modulating (SPM) interferometer to realize real-time surface profile measurement is proposed, and its measuring principle is analyzed theoretically. In the SPM interferometer, the interference signal is detected by a high speed image sensor based on a low-speed CCD and a signal processing circuit is used to obtain the phase of each point on the surface. Therefore, the surface profile can be measured real time. The experiments measuring the surface profile of a wedge-shaped optical flat show that the measurement time of the SPM interferometer is less than $10 \mathrm{~ms}$, the repetitive measurement accuracy is $5.2 \mathrm{~nm}$. The experimental results confirm the validity of the SPM interferometer, and the merits of the interferometer is simple structure, high measurement accuracy.
\end{abstract}

Keywords: real-time measurement, surface profile, SPM interferometer.

\section{Introduction}

Surface profile is one of the most important features of objects, and it is the key factor to guarantee and enhance the properties of mechanical, electronic and optical devices. Recently, the surface profile measurement technique becomes a research spot in the field of measurement [1-6]. There exist two methods in the surface profile measurement. One is contact measurement, and the other is non-contact. There exist some problems in the contact measurement, such as low accuracy and easy damage to the surface, etc. The non-contact measurement includes capacitance method, optical interference method, and scanning electronic microscope method, etc. [7-10]. In these 
methods, the optical interference method is intensively researched for the merits of high accuracy, high sensitivity, and whole field of view [11,12]. And it has been widely used in industry.

The high accuracy surface profile interferometry measurements include heterodyne interferometric method, phase-shifting interferometric method, and sinusoidally phase modulating (SPM) interferometry. The heterodyne interferometry need to shift the optical frequency accurately, which is very difficult. The phase-shifting interferometry has some faults, such as inaccurate phase-shifting, strong noise, low resistance to environmental disturbance, and complex structure, etc. Since among the characteristics of the SPM interferometry are simple phase-shifting, high accuracy, and excellent resistance to disturbance, etc., it becomes an important surface profile measurement technique [16-20]. However, at present in the SPM interferometry also exist some faults, such as bad performance in real-time, complex circuit, etc. [21]. In this paper, we propose a new method to realize the real-time surface profile measurement by using the sinusoidal phase modulating interferometer, and analyze the measuring principle theoretically. In this SPM interferometer, a high speed image sensor based on a low speed CCD is used to detect an interference signal, and a designed circuit is used to process the phase-demodulation of the interference signal to get the phase distribution of each point on the surface. According to the phase distribution, the surface profile can be obtained. And we utilize this SPM interferometer to measure the surface profile of a wedge-shaped optical flat. The experimental results confirm the validity of this SPM interferometer.

\section{Principle}

The setup of a SPM He-Ne laser interferometer for real-time 2-D surface profile measurement is shown in Fig. 1, which consists of the optical and electrical systems. A Twyman-Green interferometer is used as the optical system. After being collimated by the lens and split by the beam splitter BS, a He-Ne laser beam is split into two interference beams. One beam is reflected by a mirror and serves as a reference beam, the other is reflected by an object and serves as an object beam. The two beams interfere to form the interference signal. And this signal is imaged onto a CCD image sensor. The reference beam is phase modulated with the sinusoidally vibrating mirror which is driven by a piezoelectric transducer (PZT) attached to the back of the mirror. The interference signal is detected by a high speed image sensor based on a low speed CCD (including a special drive circuit and noise-removal circuit of CCD). After phase demodulated real-time by a signal processing circuit to the CCD output video signal, the phase distribution of the measured surface can be obtained. Therefore, we can obtain the surface profile.

The processing circuit is shown in Fig. 2, which mainly consists of a real-time phase-demodulation processing circuit, and time sequential circuit. The real-time phase-demodulation processing circuit is made up of a calculator, filter, and amplifier. 


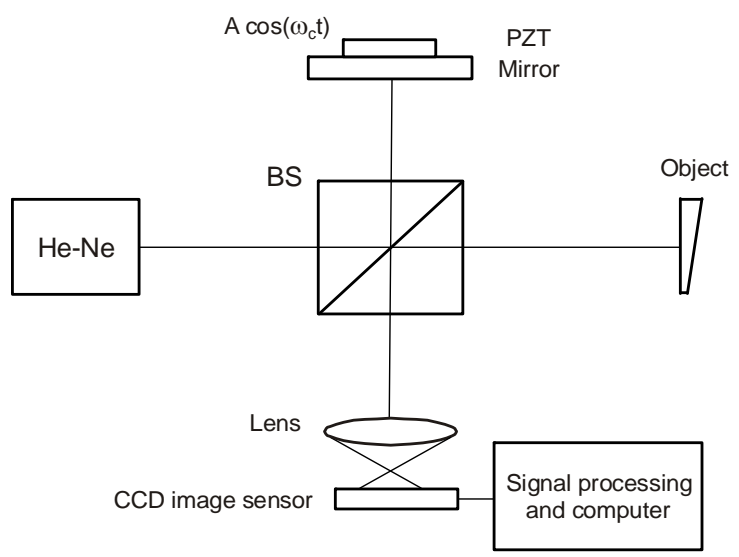

Fig. 1. Experimental setup of a SPM He-Ne laser interferometer for real-time surface profile measurement.

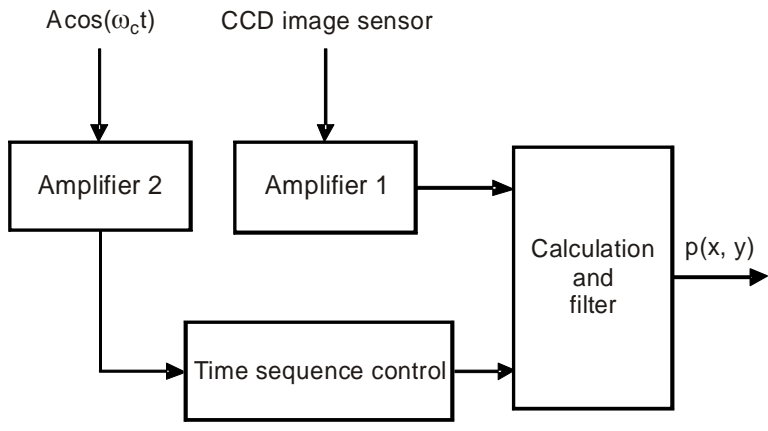

Fig. 2. The signal processing circuit.

Under the control of the time sequential circuit, the video signal is phase demodulated by the real-time phase-demodulation processing circuit, and the phase distribution can be obtained. According to the phase distribution of the surface, we can obtain the surface profile.

The modulated voltage signal $V(t)$ acted on the PZT is given by

$$
V(T)=A \cos \left(\omega_{c} t\right)
$$

where $A$ is the amplitude, $\omega_{c}$ the angular frequency. The interference signal accepted by CCD can be given by [21]

$$
s(x, y, t)=s_{1}(x, y)+s_{0}(x, y) \cos \left[z(x, y) \cos \left(\omega_{c} t\right)+\alpha_{0}+\alpha_{r}(x, y)\right]
$$

where $s_{1}$ is the dc component of the interference signal, $s_{0}$ - the amplitude of ac component, $z=4 \pi A / \lambda, \lambda$ - the wavelength of the He-Ne laser, $x$ and $y$-coordinates 
of the measured surface; $\alpha_{0}$ is the phase change of the interference signal when the mirror is still and it is determined by the optical path difference between the two interference arms $2 D_{0}$. It can be given by

$$
\alpha_{0}=\frac{4 \pi}{\lambda} D_{0}
$$

$\alpha_{r}(x, y)$ is the phase change of the interference signal arising from the measured surface profile, and it can be given by

$$
\alpha_{r}(x, y)=\frac{4 \pi}{\lambda_{0}} r(x, y)
$$

where $r(x, y)$ is the surface profile of any point on the measured surface.

Expanding Eq. (2) and neglecting the dc component, we have [22]

$$
\begin{aligned}
s(x, y, t)= & s_{0}\left\{\cos [\alpha(x, y)]\left[J_{0}(z)-2 J_{2}(z) \cos \left(2 \omega_{c} t\right)+\ldots\right]\right. \\
& \left.-\sin [\alpha(x, y)]\left[2 J_{1}(z) \cos \left(\omega_{c} t\right)-2 J_{3}(z) \cos \left(\omega_{c} t\right)+\ldots\right]\right\}
\end{aligned}
$$

where $\alpha(x, y)=\alpha_{0}+\alpha_{r}(x, y)$, and $J_{n}(z)$ is the $n$-th order Bessel function. The signal $s(x, y, t)$ and modulation voltage signal $A \cos \left(\omega_{c} t\right)$ are amplified by amplifier 1 and 2 , respectively. The two amplified signals are phase demodulated and filtered, and we obtain the signal $p(x, y)$

$$
p(x, y)=K_{A} K_{m} K_{L} S_{0} A J_{1}(z) \sin [\alpha(x, y)]
$$

where $K_{A}=K_{1} K_{2} ; K_{1}$ and $K_{2}$ are the gains of the amplifier, corresponding to amplifier 1 and 2, respectively; $K_{m}$ is the coefficient of calculation circuit, and $K_{L}$ is the gain of the filter. According to the above equation, and near the position $\alpha_{r}(x, y)=2 n \pi \pm \pi / 2$ $(n=0,1,2, \ldots)$, we can obtain

$$
\alpha(x, y)=\alpha_{0}+\alpha_{r} \approx \frac{p(x, y)}{K}
$$

where the system conversion coefficient $K=K_{A} K_{m} K_{L} s_{0} A J_{1}(z)$. Neglecting the dc component $\alpha_{0}$, we can obtain

$$
r(x, y)=\alpha(x, y) \frac{\lambda_{0}}{4 \pi}
$$

Near the position $\alpha(x, y)= \pm \pi / 2$, the signal $p(x, y)$ is proportional to the surface profile $r(x, y)$. According to Eq. (6), the span of the phase $\alpha(x, y)$ locates between 
$-\pi / 2$ and $+\pi / 2$, therefore, the measured range of $r(x, y)$ is $\lambda_{0} / 4$. Therefore, if we obtain the signal $p(x, y)$, the surface profile can be obtained.

According to above description, the high speed image sensor based on a low speed CCD can convert the interference signal into the original video signal by the special drive circuit. The noise of the original video signal can be eliminated, and we obtain the video signal. Under the control of the time sequential circuit, the real-time signal processing circuit will amplify, calculate, and filter the video signal. Therefore, we can obtain the signal $p(x, y)$. According to Eq. (8), the surface profile can be obtained real time.

\section{Experiments}

The home-made high speed image sensor based on a low speed CCD is served as an electro-photonic detector. The resolution is $30 \times 30$, the frame rate $800 \mathrm{frame} / \mathrm{second}$. The experimental setup is shown in Fig. 1. The light source is a He-Ne laser with the wavelength $632.8 \mathrm{~nm}$ and output power $8 \mathrm{~mW}$. The measured object is awedge-shaped optical flat which can cancel the disturbance of the back reflection light. The light path difference $2 D_{0}$ between the two interference arms is $6 \mathrm{~cm}$. The gain $K_{1}$ of amplifier1 is 60.2, the gain $K_{2}$ of amplifier is 288.6, the coefficient of a multiplier $K_{m}$ equals $5 \times 10^{-5} 1 / \mathrm{mV}$. In this experiment, we choose a 4-level low-pass, low-energy filter with the cut-off frequency $100 \mathrm{~Hz}$ and the gain $K_{L}=100$. The modulated voltage signal frequency acted on PZT is $398 \mathrm{~Hz}$, and the modulation voltage is $500 \mathrm{mV}$. The sinusoidal phase modulation depth is 2.58 . The measured ac component $s_{0}$ is $1.452 \mathrm{~mW}$. The system conversion coefficient $K=2.351 \times 10^{3} \mathrm{mV} / \mathrm{rad}$.

According to the detective signal $p(x, y)$, the displacement curve can be measured real-time. In order to enhance the measurement accuracy, we acquire 8 maps sequentially, and calculate the mean value of each point. The measured surface profile is shown in Figs. 3a and 3b, while the time interval is a few minutes between the two maps. The $x$ and $y$ are the position axis in units of $5 \mu \mathrm{m}$, while $z$ axis means the roughness in the units of nanometer. The repetitive measurement accuracy of
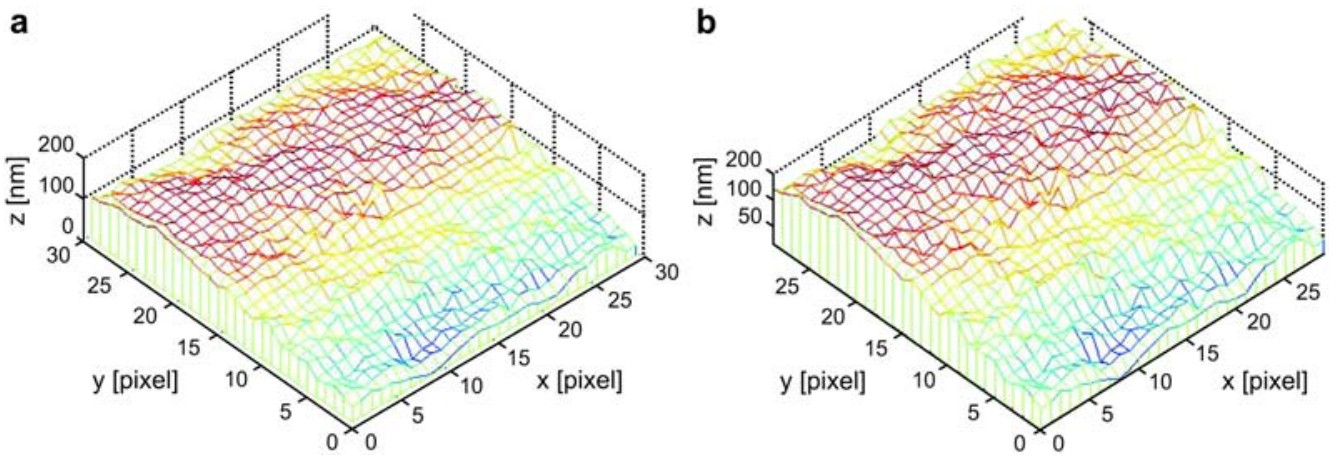

Fig. 3. The measured surface profile of the wedge-shaped (a), the measured surface profile after a time interval of a few minutes with a (b). 

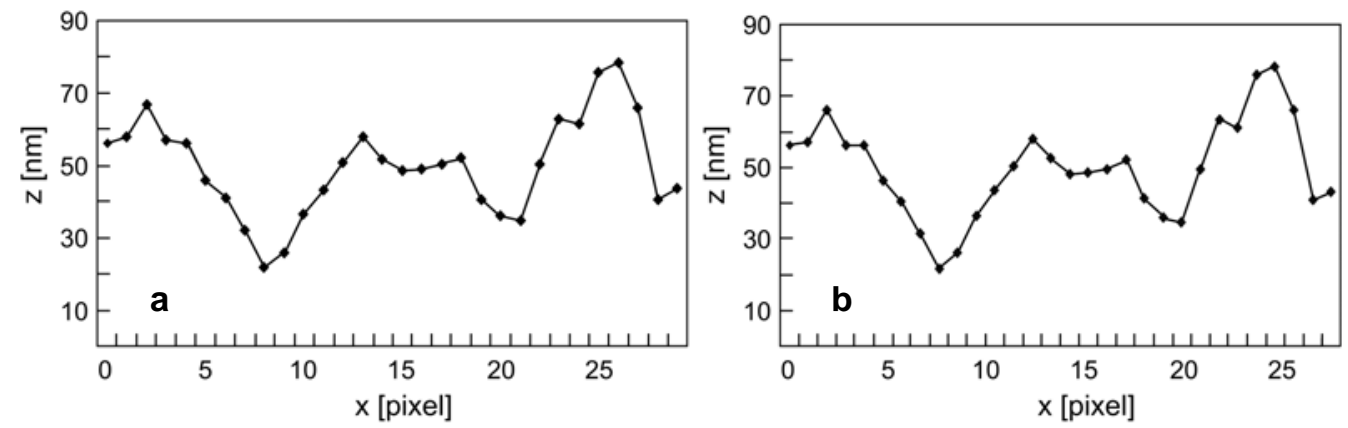

Fig. 4. Surface profile of the wedge-shaped optical flat along the $x$ axis (a), measured result after an interval of a few minutes (b).
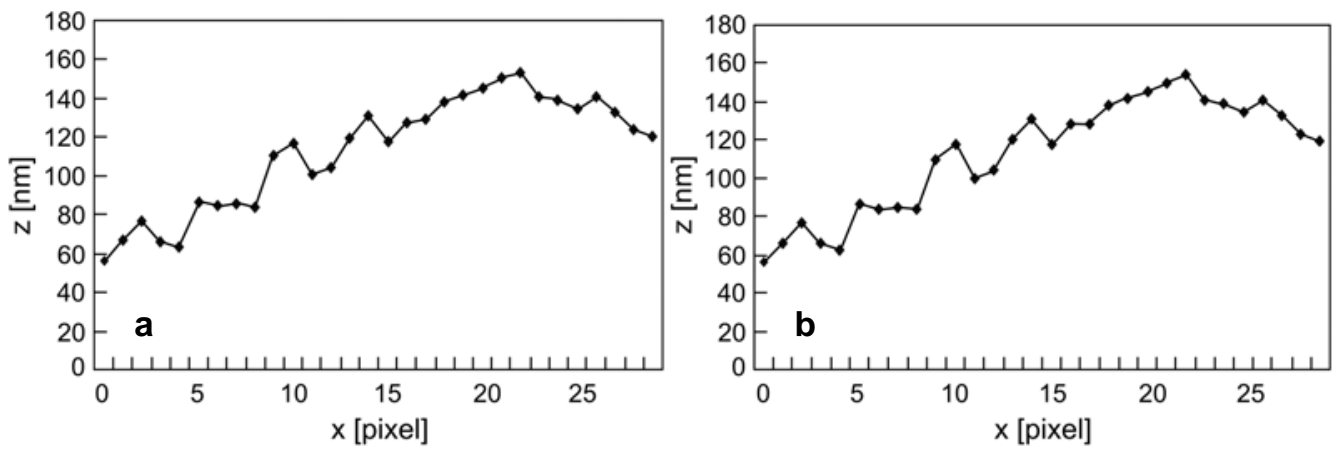

Fig. 5. Surface profile of the wedge-shaped optical flat along the $y$ axis (a), measured result after an interval of a few minutes (b).

Fig. $3 \mathbf{a}$ is $5.2 \mathrm{~nm}$. The related coefficient of Figs. $3 \mathbf{a}$ and $3 \mathbf{b}$ is 0.9826 , and the maximum difference value is $3.6 \mathrm{~nm}$. The read-out time of a pixel is $0.125 \mu \mathrm{m}$, and the retardation time of the circuits is less than 200 ns. The time for phase-demodulation and calculation is less than $8 \mathrm{~ms}$. Therefore the measurement time is less than $10 \mathrm{~ms}$.

In order to analyze the measurement accuracy clearly, we can select a row or column from Fig. 3a randomly to analyze the accuracy. Figures $4 \mathbf{a}$ and $4 \mathbf{b}$ show the maps of the first row in the direction of $x$ axis of Figs. 3a and 3b, respectively. The repetitive measurement accuracy of Fig. $4 \mathbf{a}$ is $3.681 \mathrm{~nm}$, while that of Fig. $4 \mathbf{b}$ is $3.653 \mathrm{~nm}$. In the same way, we select the first column in the direction of $y$ axis of Figs. $3 \mathbf{a}$ and $3 \mathbf{b}$, results are shown in Figs. $5 \mathbf{a}$ and $5 \mathbf{b}$. The repetitive measurement accuracy of Fig. $5 \mathbf{a}$ is $3.852 \mathrm{~nm}$, while that of Fig. $4 \mathbf{b}$ is $3.925 \mathrm{~nm}$. The related coefficient of Figs. $4 \mathbf{a}$ and $4 \mathbf{b}$ is 0.9821 , while that of Figs. $5 \mathbf{a}$ and $5 \mathbf{b}$ is 0.9825.

\section{Conclusions}

In this paper, a sinusoidal phase modulating (SPM) interferometer to realize real-time surface profile measurement is proposed, and its measurement principle is analyzed 
theoretically. In the SPM interferometer, the interference signal is detected by a high speed image sensor based on a low-speed CCD and a signal processing circuit is used to obtain the phase of each point on the surface. Therefore, the surface profile can be measured real time. The experiments measuring the surface profile of a wedge-shaped optical flat show that the measurement time of the SPM interferometer is less than $10 \mathrm{~ms}$, the repetitive measurement accuracy is $5.2 \mathrm{~nm}$. The experimental results confirm the validity of the SPM interferometer, and the merits of the interferometer are simple structure and high measurement accuracy.

\section{References}

[1] Tay C.J., Quan C., Fu Y., Cen L.J., Shang H.M., Surface profile measurement of low-frequency vibrating objects using temporal analysis of fringe pattern, Optics and Laser Technology 36(6), 2004, pp. 471-6.

[2] Shinozaki R., Sasaki O., Suzuki T., Fast scanning method for one-dimensional surface profile measurement by detecting angular deflection of a laser beam, Applied Optics 43(21), 2004, pp. 4157-63.

[3] Yin S., Li J., Song M., Surface profile measurement using a unique microtube-based system, Optics Communications 168(1-4), 1999, pp. 1-6.

[4] Shengfeng Lu, Yongsheng Gao, Tiebang Xie, Feng Xie, Jiang X.Q., Zhu Li, Fangmin Wang, A novel contact/non-contact hybrid measurement system for surface topography characterization, International Journal of Machine Tools and Manufacture 41(13-14), 2001, pp. 2001-9.

[5] Yокота M, Asaka A, Yoshino T., Stabilization improvements of laser-diode closed-loop heterodyne phase-shifting interferometer for surface profile measurement, Applied Optics 42(10), 2003, pp. 1805-8.

[6] TAY C.J., QuAn C., Fu Y., HuAng Y., Instantaneous velocity displacement and contour measurement by use of shadow moiré and temporal wavelet analysis, Applied Optics 43(21), 2004, pp. 4164-71.

[7] Chen F., Brown G.M., Song M., Overview of three-dimensional shape measurement using optical methods, Optical Engineering 39(1), 2002, pp. 10-22.

[8] Bennett J.M., Jahanmir J., Podlesny J.C., Balter T.L., Hobbs D.T., Scanning force microscope as a tool for studying optical surface, Applied Optics 34(1), 1995, pp. 213-30.

[9] Binning G., Rohrer H., Gerber C., Weibel W., Surface studies by scanning tunneling microscope, Physical Review Letters 49(1), 1982, pp. 57-61.

[10] Binning G., Quate C.F., Gerber C., Atomic force microscopy, Physical Review Letters 56(9), 1986, pp. 930-3.

[11] Jin G.F., Li J.Z., Laser Surveying, Science Publishing House, Beijing 1998, pp. 5-300.

[12] YAng G., The Modern Optical Measurement Technology, The Jiang University Press, Hangzhou 1997, pp. 9-118.

[13] Polster H.D., Pastor J., Scott R., Crane R., Langenbeck P.H., Pilston R., Steinberg G., New developments in interferometry, Applied Optics 8(3), 1969, p. 521.

[14] Bruning J.H., Herriott D.R., Gallagher J.E., Rosenfeld D.P., White A.D., Brangaccio D.J., Digital wavefront measuring interferometer for testing optical surfaces and lenses, Applied Optics 13(11), 1974, pp. 2693-703.

[15] Sasaki O., Окаzакі H., Sinusoidal phase modulating interferometry for surface profile measurement, Applied Optics 25(18), 1986, pp. 3137-40.

[16] Luan Z., Liu L., Liu D, Teng S., Double shearing wavefront testing, Acta Optica Sinica 24(10), 2004, pp. 1417-20.

[17] SasaKi O., OKamura T., NAKAmura T., Sinusoidal phase modulating Fizeau interferometer, Applied Optics 29(4), 1990, pp. 512-5. 
[18] Takamasa Suzuki, Osami Sasaki, Shuich Takayama, Takeo Maruyama, Real-time displacement measurement using synchronous detectionin a sinusoidal phase modulating interferometer, Optical Engineering 32(5), 1993, pp. 1033-7.

[19] Li D., WANG X.Z., LIU Y.M., Fiver optic interferometer for real-time wide-range distance measurements with ANN, Acta Photonica Sinica 34(6), 2005, pp. 865-8.

[20] Wang Xuefeng, Wang Xiangzhao, Yu Danyang, Qian Feng, Lu Hongbin, Photothermal modulation laser diode interferometer insensitive to external disturbances, Acta Optica Sinica 21(11), 2001, pp. 1368-71.

[21] Takamasa Suzuki, Osami Sasaki, Jinsaku Kaneda, Takeo Maruyama, Real-time two-dimensional surface profile measurement in a sinusoidal phase-modulating laser diode interferometer, Optical Engineering 33(8), 1994, pp. 2754-9.

[22] Takamasa Suzuki, Osami Sasaki, Takeo Maruyama, Phase locked laser diode interferometry for surface profile measurement, Applied Optics 28(20), 1989, pp. 4407-10.

Received August 26, 2007 